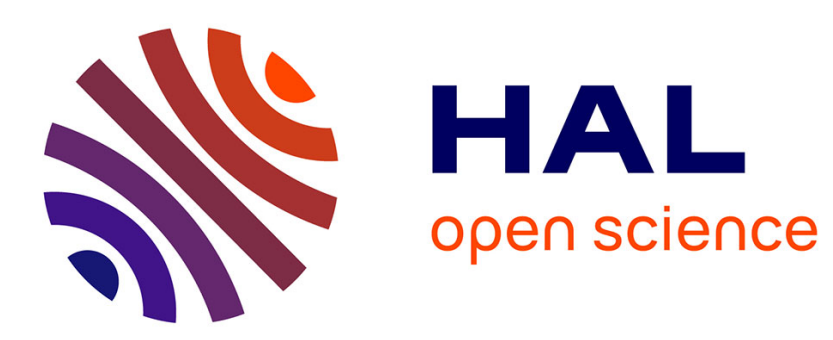

\title{
Anti-scattering effect analyzed with an exact theory of light scattering from rough multilayers
}

\author{
G. Soriano, Myriam Zerrad, C. Amra
}

\section{To cite this version:}

G. Soriano, Myriam Zerrad, C. Amra. Anti-scattering effect analyzed with an exact theory of light scattering from rough multilayers. Optics Letters, 2019, 44 (18), pp.4455. 10.1364/OL.44.004455 . hal-02410346

\section{HAL Id: hal-02410346 \\ https://hal.science/hal-02410346}

Submitted on 12 May 2020

HAL is a multi-disciplinary open access archive for the deposit and dissemination of scientific research documents, whether they are published or not. The documents may come from teaching and research institutions in France or abroad, or from public or private research centers.
L'archive ouverte pluridisciplinaire HAL, est destinée au dépôt et à la diffusion de documents scientifiques de niveau recherche, publiés ou non, émanant des établissements d'enseignement et de recherche français ou étrangers, des laboratoires publics ou privés. 


\title{
Anti-scattering effect analyzed with exact theory of light scattering from rough multilayers
}

\author{
G. SoriAno ${ }^{1,}$, M. ZERRAD ${ }^{1}$, AND C.AMra ${ }^{1}$ \\ ${ }^{1}$ Aix-Marseille Université, CNRS, Centrale Marseille, Institut Fresnel, UMR 7249, 13013 Marseille, France \\ *Corresponding author: gabriel.soriano@fresnel.fr
}

Compiled August 13, 2019

First-order theories of light scattering previously revealed the existence of anti-scattering effects in optical multilayers. Here we present an exact electromagnetic theory able to complete the scattering analysis when first-order scattering is cancelled. The theory is valid for arbitrary rough multilayers. (๑) 2019 Optical Society of America

http://dx.doi.org/10.1364/ao.XX.XXXXXX

\section{INTRODUCTION}

Light scattering from slightly rough multilayers [1-5] has been extensively studied in the field of optical interference coatings $[6,7]$. Such planar multilayers most often consist of dielectric materials produced with vacuum deposition technologies (Electron Beam Deposition, Ion Assisted Deposition, Dual Ion Beam Sputtering, Magnetron Sputtering) which allow materials to grow in amorphous thin film form on fused silica substrates and others. It is well known $[2,3,5]$ that in the optical band-pass of spatial frequencies [8], substrate roughness is replicated from one interface to another within these multilayers, and that this replication effect creates a roughness threshold which is most often responsible for the major scattering contribution. Since polishing techniques have allowed to reduce substrate roughness to values down to a fraction of nanometer, all interfaces of optical coatings are slightly rough and this is the reason why first-order electromagnetic theories have shown great success when analyzing angular and wavelength scattering patterns from these components. Numerous results can be found [2, 3, 5] which emphasize an excellent agreement between theory and experiment, even for complex coatings involving hundreds of layers.

However there are a few situations where first-order theory must be completed even though the surfaces are slightly rough. One iconic situation is that of the anti-scattering effect that was shown $[9,10]$ to occur at specific angles or wavelengths, due to destructive interferences between waves scattered from different fully correlated surfaces. In this case the first-order scattering is perfectly cancelled while low-level signals can still be measured and must be taken into account; indeed for an increasing number of applications (complex micro-filters for space multiplexing, mirrors for gravitational-wave detection), the energy balance (including reflection, transmission, absorption and scattering) must be known with an absolute accuracy of one ppm (1ppm = $10^{-6}$ ). Hence facing this difficulty requires to have at disposal a higher-order theory [11-13], or rather an exact electromagnetic theory [14, 15], able to predict light scattering from arbitrary rough multilayers.

Though different formalisms [16-18] were developed to take account of arbitrary roughness at one single (uncoated) surface, until now only a few of them have addressed the case of multilayers [19]. This is the goal of this paper to present an exact electromagnetic theory based on an extension of the boundary integral equation (BIE) method [18, 20-22]. Numerical calculation is given in two-dimensional scattering configuration [23] for single layers involving a set of roughness parameters [24] allowing the anti-scattering effect to occur, and the results are focused on a comparison with first-order theory.

\section{THE ROUGH LAYER BOUNDARY INTEGRAL FOR- MALISM}

We consider a three homogeneous media problem where the layer $\Omega_{2}$ is bounded by two non-intersecting rough surfaces $\Sigma_{n}$ with equations $z=z_{n}+h_{n}(\mathbf{r})$ for $n=1,2$ in the Cartesian coordinates $(x, y, z)$, and denoting $(x, y)=\mathbf{r}$. It is assumed that the two boundaries don't overlap, that is $z_{1}+h_{1}(\mathbf{r})>z_{2}+$ $h_{2}(\mathbf{r})$ for all $\mathbf{r}$. The layer is enlightened from superstrate $\Omega_{1}$ : $z>z_{1}+h_{1}(\mathbf{r})$ through interface $\Sigma_{1}$. Wavelength in vacuum is denoted $\lambda_{0}$, so that angular frequency is $\omega=2 \pi c / \lambda_{0}$. Surface $\Sigma_{2}$ interfaces the layer from substrate $\Omega_{3}$. Electromagnetical parameters at wavelength $\lambda_{0}$ are denoted $\left(\varepsilon_{m}, \mu_{m}\right)$ with $m=1$ in the superstrate, $m=2$ in the layer and $m=3$ in the substrate.

Assuming an $e^{-i \omega t}$ implicit time-dependency, the electromagnetic field $(\mathbf{E}, \mathbf{H})$ satisfies in domain $\Omega_{m}$ the time-harmonic Maxwell's equations curl $\mathbf{E}=+i \omega \mu_{m} \mathbf{H}$ and curl $\mathbf{H}=-i \omega \varepsilon_{m} \mathbf{E}$. The tangential components of the field $\hat{\mathbf{n}}_{p} \times \mathbf{E}$ and $\hat{\mathbf{n}}_{p} \times \mathbf{H}$ on both interfaces $p=1,2$ are continuous. $\hat{\mathbf{n}}_{p}$ denotes the unit normal vector, oriented from $\Omega_{p+1}$ toward $\Omega_{p}$. This field also satisfies an outgoing wave condition in the substrate $\Omega_{3}$. The incident field $\left(\mathbf{E}^{i n c}, \mathbf{H}^{i n c}\right)$ satisfies the Maxwell's equations for $m=1$, but in the whole space. It writes as a sum of downwarddirected plane waves

$$
\mathbf{E}^{i n c}(\mathbf{r}, z)=\int_{\mathbb{R}^{2}} \mathbf{E}_{1}^{0-}(\mathbf{k}) e^{i\left(\mathbf{k} \cdot \mathbf{r}-q_{1} z\right)} d \mathbf{k}
$$

with $\mathbf{k}^{2}+q_{1}^{2}=\omega^{2} \varepsilon_{1} \mu_{1}$ and $0 \leq \arg q_{1} \leq \pi / 2$. The scattered 


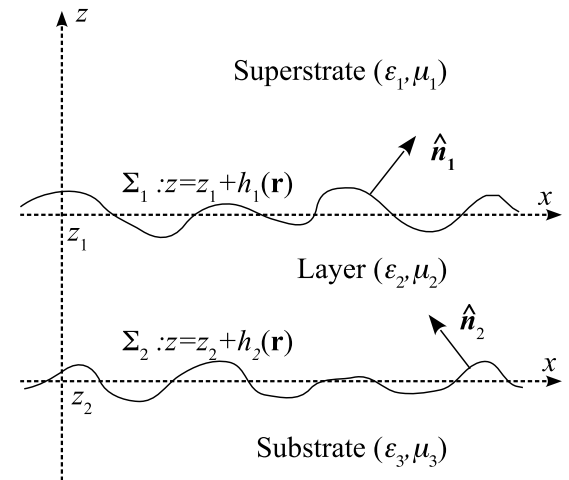

Fig. 1. Geometry of the problem

field $\left(\mathbf{E}-\mathbf{E}^{i n c}, \mathbf{H}-\mathbf{H}^{i n c}\right)$ satisfies an outgoing wave condition in the superstrate.

The reference field $\left(\mathbf{E}^{0}, \mathbf{H}^{0}\right)$ corresponds to the field $(\mathbf{E}, \mathbf{H})$ in the case where the layer surfaces are two parallel planes $z=z_{1}$ and $z=z_{2}$. The plane waves decompositions of the reference field in the three media write

$$
\int_{\mathbb{R}^{2}}\left\{\mathbf{E}_{m}^{0+}(\mathbf{k}) e^{i\left(\mathbf{k} \cdot \mathbf{r}+q_{m} z\right)}+\mathbf{E}_{m}^{0-}(\mathbf{k}) e^{i\left(\mathbf{k} \cdot \mathbf{r}-q_{m} z\right)}\right\} d \mathbf{k}=\mathbf{E}_{m}^{0}(\mathbf{r}, z)
$$

with $\mathbf{k}^{2}+q_{m}^{2}=\omega^{2} \varepsilon_{m} \mu_{m}$ and $0 \leq \arg q_{m} \leq \pi / 2$. Outgoing wave condition in the substrate leads to $E_{3}^{0+}(\mathbf{k})=\mathbf{0}$ for all $\mathbf{k}$. In eq. (2), those three plane wave decompositions are extended to the whole space to define the three fields $\left(\mathbf{E}_{m}^{0}, \mathbf{H}_{m}^{0}\right)$.

Now, for rough interfaces, the tangential components of the field $\hat{\mathbf{n}}_{p} \times \mathbf{E}$ and $\hat{\mathbf{n}}_{p} \times \mathbf{H}$, cast into the vector form

$$
X=\left[\begin{array}{c}
\hat{\mathbf{n}}_{1} \times \mathbf{E} \\
\hat{\mathbf{n}}_{1} \times \mathbf{H} \\
\hat{\mathbf{n}}_{2} \times \mathbf{E} \\
\hat{\mathbf{n}}_{2} \times \mathbf{H}
\end{array}\right]
$$

satisfy the linear system

$$
A(X-Y)=C(Z-Y)
$$

of coupled boundary integral equations. The two vectors

$$
Y=\left[\begin{array}{c}
\hat{\mathbf{n}}_{1} \times \mathbf{E}_{1}^{0} \\
\hat{\mathbf{n}}_{1} \times \mathbf{H}_{1}^{0} \\
\hat{\mathbf{n}}_{2} \times \mathbf{E}_{2}^{0} \\
\hat{\mathbf{n}}_{2} \times \mathbf{H}_{2}^{0}
\end{array}\right] \quad Z=\left[\begin{array}{c}
\hat{\mathbf{n}}_{1} \times \mathbf{E}_{2}^{0} \\
\hat{\mathbf{n}}_{1} \times \mathbf{H}_{2}^{0} \\
\hat{\mathbf{n}}_{2} \times \mathbf{E}_{3}^{0} \\
\hat{\mathbf{n}}_{2} \times \mathbf{H}_{3}^{0}
\end{array}\right]
$$

are determined from the reference field on the rough boundaries. Matrix $A$ writes

$$
A=\left[\begin{array}{cccc}
\frac{1}{2}-\mathcal{K}_{1}^{11} & -\mu_{1} \mathcal{T}_{1}^{11} & 0 & 0 \\
\frac{1}{2}+\mathcal{K}_{2}^{11} & +\mu_{2} \mathcal{T}_{2}^{11} & -\mathcal{K}_{2}^{12} & -\mu_{2} \mathcal{T}_{2}^{12} \\
+\mathcal{K}_{2}^{21} & +\mu_{2} \mathcal{T}_{2}^{21} & \frac{1}{2}-\mathcal{K}_{2}^{22} & -\mu_{2} \mathcal{T}_{2}^{22} \\
0 & 0 & \frac{1}{2}+\mathcal{K}_{3}^{22} & +\mu_{3} \mathcal{T}_{3}^{22}
\end{array}\right]
$$

introducing (notations are derived from [25] and generalized to layered media) the EFIE operator

$$
\mathcal{T}_{m}^{n p} \mathbf{j}\left(\mathbf{R}_{n}\right)=\hat{\mathbf{n}}_{n} \times \int_{\Sigma_{p}} i \omega \overline{\mathbf{G}}_{m}\left(\mathbf{R}_{n}-\mathbf{R}_{p}\right) \cdot \mathbf{j}\left(\mathbf{R}_{p}\right) d S_{p}
$$

and the MFIE operator

$$
\mathcal{K}_{m}^{n p} \mathbf{j}\left(\mathbf{R}_{n}\right)=\hat{\mathbf{n}}_{n} \times \int_{\Sigma_{p}} \operatorname{curl} \overline{\mathbf{G}}_{m}\left(\mathbf{R}_{n}-\mathbf{R}_{p}\right) \cdot \mathbf{j}\left(\mathbf{R}_{p}\right) d S_{p}
$$

with $\mathbf{j}$ a tangential vector field and for two point $\mathbf{R}_{n}$ and $\mathbf{R}_{p}$ on interfaces $\Sigma_{n}$ and $\Sigma_{p}$, respectively. Those operators involve the free space dyadic Green's functions $\overline{\mathbf{G}}_{m}(\mathbf{R})=$ $\left(\overline{\mathbf{I}}+K_{m}^{-2}\right.$ grad div $) G_{m}(\mathbf{R})$. For passive media $m=1,2,3$, the wavenumbers $K_{m}$ satisfy $K_{m}^{2}=(2 \pi / \lambda)^{2} \varepsilon_{m} \mu_{m}$ with $0 \leq$ $\arg K_{m} \leq \pi / 2$. The scalar Green's functions are driven by equations (div grad $\left.-K_{m}^{2}\right) G_{m}(\mathbf{R})=-\delta(\mathbf{R})$ and radiation condition. Matrix $C$

$$
C=\left[\begin{array}{cccc}
0 & 0 & 0 & 0 \\
\frac{1}{2}+\mathcal{K}_{2}^{11} & +\mu_{2} \mathcal{T}_{2}^{11} & 0 & 0 \\
+\mathcal{K}_{2}^{21} & +\mu_{2} \mathcal{T}_{2}^{21} & 0 & 0 \\
0 & 0 & \frac{1}{2}+\mathcal{K}_{3}^{22} & +\mu_{3} \mathcal{T}_{3}^{22}
\end{array}\right]
$$

is a sparsified version of matrix $A$. Such a theory can easily be extended to structures with an arbitrary number of layers.

Then, the scattered electric field writes in the $z>z_{1}+\max h_{1}$ region of the superstrate as the sum of plane waves:

$$
\left(\mathbf{E}-\mathbf{E}^{i n c}\right)(\mathbf{r}, z)=\int_{\mathbb{R}^{2}} \mathbf{E}^{+}(\mathbf{k}) e^{i\left(\mathbf{k} \cdot \mathbf{r}+q_{1} z\right)} d \mathbf{k}
$$

Their amplitude is related to the tangential components of the fields on $\Sigma_{1}$ through expression:

$$
\begin{aligned}
\mathbf{E}^{+}(\mathbf{k})=+\frac{\mathbf{K}_{1}}{8 \pi^{2} q_{1}} \times \int_{\Sigma_{1}} & \left\{\frac{\mathbf{K}_{1}}{\omega \varepsilon_{1}} \times\left(\hat{\mathbf{n}}_{1} \times \mathbf{H}\right)\right. \\
& \left.-\hat{\mathbf{n}}_{1} \times \mathbf{E}\right\} e^{-i \mathbf{K}_{1} \cdot \mathbf{R}_{1}} d S_{1}
\end{aligned}
$$

with $\mathbf{K}_{1}=\mathbf{k}+q_{1} \hat{\mathbf{z}}$ the upward-directed wavevector.

Finally, this electric field formulation is turned into a magnetic field formulation by substituying $\mathbf{E} \leftrightarrow \mathbf{H}$ and $\mu \leftrightarrow-\varepsilon$ in eqs. (1)-(6) and (9)-(11).

\section{NUMERICAL CALCULATION}

Because numerical calculation is highly time consuming, the BIE theory (4) was implemented for one-dimensional (1D) surfaces, and discretized with the method of moments [23]. A first step consisted in a direct comparison with first-order theory (SPM). For that we considered a sample which is a non-absorbing highindex quarter-wave layer $\left(n_{H} e_{H}=\lambda_{0} / 4\right)$ at the illumination wavelength $\lambda_{0}=632.8 \mathrm{~nm}$, with $n_{H}=2.3$ the optical index and $e_{H}$ the layer thickness. The superstrate is air $\left(n_{1}=1\right)$ and the substrate is glass $\left(n_{3}=1.52\right)$. Incident field is a Gaussian beam, centered on normal incidence $\left(i=0^{\circ}\right)$ and with $1.5^{\circ}$ divergence (tapering parameter [18] is $g=8 \mu \mathrm{m}$ ). Both surfaces have Gaussian roughness with autocorrelation length $L=300 \mathrm{~nm}$, and their cross-correlation coefficient [13] is denoted $\alpha$. In figure 2 their height root mean squares $\left(\delta_{1}=\delta_{2}=5 \mathrm{~nm}\right)$ are set identical, but the surface profiles can be fully cross-correlated $(\alpha=1)$ or totally cross-uncorrelated $(\alpha=0)$.

We observe in figure 2, where polarization is TM, a very high agreement with first-order theory, due to the low roughness-towavelength ratio. Such agreement holds in the whole angular range, and for the two extreme cases of cross-correlation. As a reminder, the cross-correlated $(\alpha=1)$ scattering takes account of interferences between the waves scattered by the two interfaces, while no interference occurs in the uncorrelated case $(\alpha=0)$. 

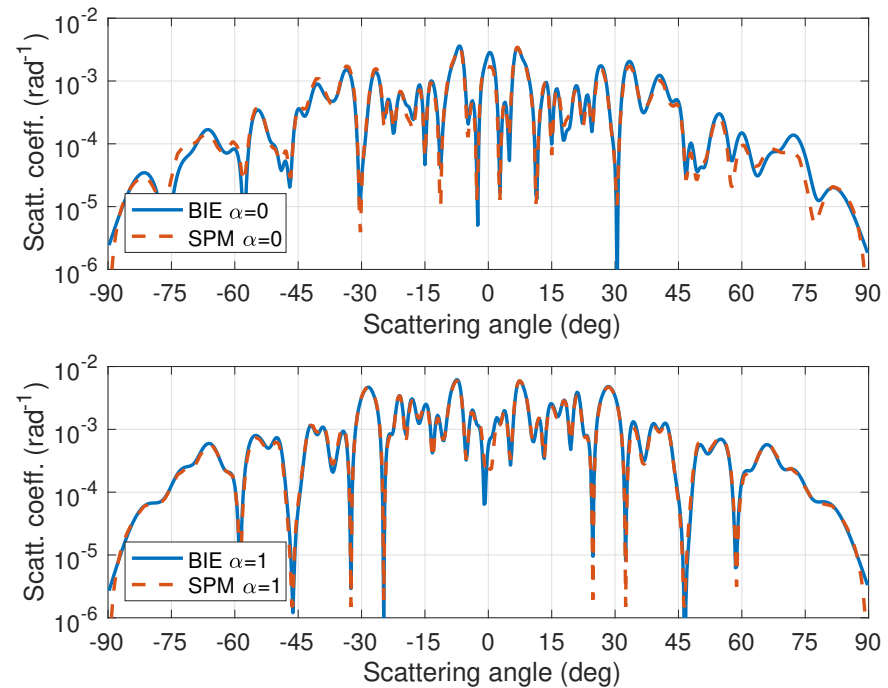

Fig. 2. Scattering diagram for a high-index quarter-wave layer at $632.8 \mathrm{~nm}$ wavelength, normal incidence and polarization TM. Roughness is $5 \mathrm{~nm}$ for both interfaces. Surface profiles are either totally uncorrelated ( $\alpha=0$, top plot) or fully correlated ( $\alpha=1$, bottom plot).

This is due to the fact that cross-correlation acts as a mutual coherence factor $[9,10]$. Hence these first results validate the comparison with first-order theory.

Now we focus the analysis on the anti-scattering effect [9] predicted with first-order theory. Actually in [9] it was shown that single layers may reveal an analytical zero of light scattering under the assumption of fully cross-correlated surfaces and specific roughness values. This effect is the result of destructive interferences between the waves scattered from surfaces $\Sigma_{1}$ and $\Sigma_{2}$, for which reason full cross-correlation is required. Furthermore, for these interferences to be destructive, the thin film should be low-index quarter-wave or high-index half-wave at the illumination wavelength $\lambda_{0}$. Eventually the roughness ratio $\delta_{1} / \delta_{2}$ of the two surfaces must satisfy a condition related to the three index materials, that is [9]:

$$
\frac{\delta_{1}}{\delta_{2}}=\frac{n_{2}^{2}}{n_{3}^{2}} \frac{n_{3}^{2}-n_{2}^{2}}{n_{2}^{2}-n_{1}^{2}}
$$

for the low-index quarter-wave layer, and

$$
\frac{\delta_{1}}{\delta_{2}}=\frac{n_{2}^{2}-n_{3}^{2}}{n_{2}^{2}-n_{1}^{2}}
$$

for the high-index half-wave layer. This last condition is given for a scattering cancellation at scattering angle $\theta=0^{\circ}$.

In Figs. 3 and 4, we considered similar samples (single layers) and used our exact theory for Monte Carlo comparison to first-order theory under normal illumination. The Monte Carlo average is performed over 32 samples. The thin film materials are non-absorbing and their real indices are given at the illumination wavelength $\lambda_{0}=632.8 \mathrm{~nm}$ by $n_{H}=2.3$ and $n_{L}=1.3$. Such indices were given in [9] for $\mathrm{ZnS}$ and $\mathrm{Na}_{3} \mathrm{AlF}_{6}$ thin film materials. Hence the optical thicknesses follow: $n_{H} e_{H}=\lambda_{0} / 2$ and $n_{L} e_{L}=\lambda_{0} / 4$ with $e$ the thickness. As previously, the (glass) substrate roughness is $\delta_{1}=5 \mathrm{~nm}$.

Figs. 3 and 4 are respectively given for the low-index quarterwave layer in TE polarization and the high-index half-wave

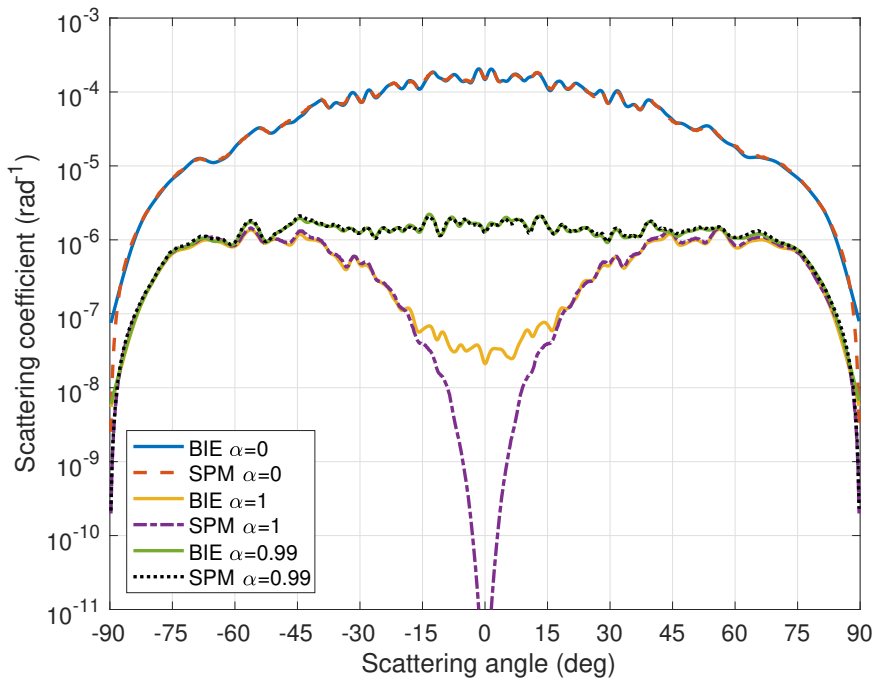

Fig. 3. Scattering diagram for a low-index quarter-wave layer at $632.8 \mathrm{~nm}$ wavelength, normal incidence and polarization TE. The layer-glass interface roughness is $5 \mathrm{~nm}$, while the air-layer interface roughness satisfies the antiscattering condition (12).

layer in TM polarization, with the roughness ratios given by Eq. (12) and Eq. (13). As in Fig. 2, for the scattering from crossuncorrelated rough profiles ( $\alpha=0$ curves on Figs. 3 and 4 ), we observe a high agreement between first-order theory and the exact calculation in the whole angular range. As for the cross-correlated ( $\alpha=1$ curves on Figs. 3 and 4 ) scattering, the agreement still remains high for most scattering angles, but it fails in the close vicinity of the specular beam $\left(\theta=0^{\circ}\right)$. Such difference was expected since first-order scattering is zero in this vicinity; hence the remaining signal is characteristic of higher order scattering. This result emphasizes the interest of an exact theory for further analysis of the anti-scattering effect.

However before conclusion it should be stressed that the analytical zero of first-order light scattering is highly sensitive to the cross-correlation coefficient around unity. Actually even a slight departure from the value $\alpha=1$ will break the interferential balance and increase the first-order signal. This is emphasized in Figs. 3 and 4 where first-order and exact scattering are also plotted for a cross-correlation value of $\alpha=0.99$. With these supplementary curves, it is clear that the first-order scattering is far from zero at $\theta=0^{\circ}$, even though the variation in crosscorrelation is only $1 \%$. Furthermore, we observe that the two theories (first-order and exact) again reveal a high agreement for $\alpha=0.99$, due to the fact that first-order scattering is again predominant. This result justifies why first-order calculation in multilayers is most often considered with cross-correlation values around 0.99 [5] (rather than 1), all the more than a quasiperfect (rather than perfect) replication of topography is more realistic.

\section{CONCLUSION}

We have developed an exact electromagnetic theory of light scattering from arbitrary rough surfaces. The theory is a discretized set of coupled boundary integral equations. However, with a modified right-hand side, it differs from the classical formulation [22]. Our approach was compared with great success to first-order theory in the whole angular range. In order to 


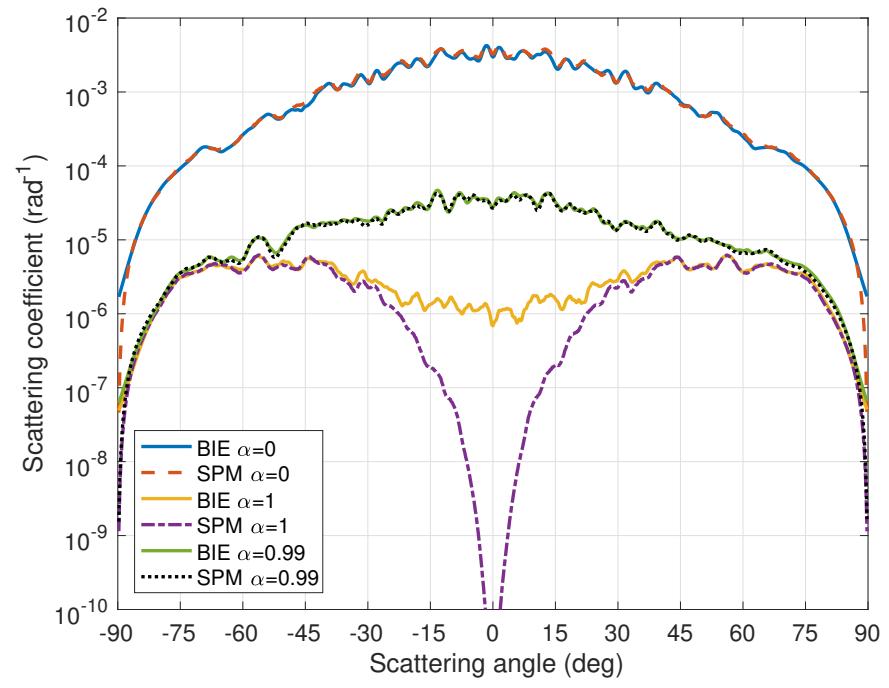

Fig. 4. Scattering diagram for a high-index half-wave layer at $632.8 \mathrm{~nm}$ wavelength, normal incidence and polarization TM. The layer-glass interface roughness is $5 \mathrm{~nm}$, while the air-layer interface roughness satisfies the antiscattering condition (13).

emphasize higher-order scattering, we analyzed anti-scattering effects predicted by first-order theory. The exact theory was able to quantify the scattering level when first-order scattering is zero. This is a key point, since new trends in optical coatings are about to take profit of the anti-scattering effect to minimize losses $[26,27]$. The exact theory might also lead, through future works, to the definition of improved anti-scattering conditions. We also quantified the sensitivity of scattering to interfaces crosscorrelation. This allows to establish a more accurate energy balance, as required in high-precision optical systems (pixellized filters for space micro-multiplexing, mirrors for detection of gravitational waves...) To conclude, this exact theory will also meet other applications in the field of radar probing of soil and moisture, cosmetics and living tissues, lighting, textiles and stationery.

\section{REFERENCES}

1. J. Elson, J. Rahn, and J. Bennett, Appl. Opt. 22, 3207 (1983).

2. C. Amra, JOSA A 11, 197 (1994).

3. C. Amra, JOSA A 11, 211 (1994).

4. S. Schröder, T. Herffurth, H. Blaschke, and A. Duparré, Appl. optics 50, C164 (2011).

5. M. Zerrad, S. Liukaityte, M. Lequime, and C. Amra, Appl. optics 55, 9680 (2016).

6. H. A. Macleod and H. A. Macleod, Thin-film optical filters (CRC press, 2010).

7. P. W. Baumeister, Optimization 10, 7 (2004).

8. C. Deumié, R. Richier, P. Dumas, and C. Amra, Appl. optics 35, 5583 (1996).

9. C. Amra, G. Albrand, and P. Roche, Appl. optics 25, 2695 (1986).

10. C. Amra, J. H. Apfel, and E. Pelletier, Appl. optics 31, 3134 (1992).

11. M. Demir, J. Johnson, and T. Zajdel, Geosci. Remote. Sensing, IEEE Transactions on 50, 3374 (2012).

12. M. Sanamzadeh, L. Tsang, J. T. Johnson, R. J. Burkholder, and S. Tan, JOSA A 34, 395 (2017).

13. J.-P. Banon, Ø. S. Hetland, and I. Simonsen, Annals Phys. 389, 352 (2018).

14. C.-H. Kuo and M. Moghaddam, IEEE Transactions on antennas propagation 54, 2917 (2006).
15. M. Sanamzadeh, L. Tsang, and J. T. Johnson, IEEE Transactions on Antennas Propag. 67, 495 (2018).

16. C. Amra, C. Grèzes-Besset, and L. Bruel, Appl. optics 32, 5492 (1993).

17. T. Elfouhaily and C. A. Guérin, Waves Random Media 14, R1 (2004).

18. L. Tsang, J. A. Kong, K. H. Ding, and C. O. Ao, Scattering of electromagnetic waves: numerical simulations, Wiley series in remote sensing (Wiley-Interscience, 2001).

19. P. Imperatore, A. lodice, M. Pastorino, and N. Pinel, Int. J. Antennas Propag. 2017 (2017).

20. D. Colton and R. Kress, Integral equation methods in scattering theory, vol. 72 (SIAM, 2013).

21. W. Chew, M. Tong, and B. Hu, Integral Equation Methods for Electromagnetic and Elastic Waves (Morgan \& Claypool, 2008).

22. N. Déchamps, N. de Beaucoudrey, C. Bourlier, and S. Toutain, JOSA A 23, 359 (2006).

23. C. Bourlier, N. Pinel, and G. Kubické, Method of moments for $2 D$ scattering problems: basic concepts and applications (John Wiley \& Sons, 2013).

24. J. A. Ogilvy, Theory of Wave Scattering from Random Rough Surfaces (Adam Hilger, Bristol, 1991).

25. G. Hsiao and R. Kleinman, Antennas Propagation, IEEE Transactions on 45, 316 (1997).

26. J. Zhang, H. Wu, H. Jiao, S. Schröder, M. Trost, Z. Wang, and X. Cheng, Opt. letters 42, 5046 (2017).

27. J. Zhang, H. Wu, I. V. Kozhevnikov, S. Shi, X. Cheng, and Z. Wang, Opt. express 27, 15262 (2019). 


\section{FULL REFERENCES}

1. J. Elson, J. Rahn, and J. Bennett, "Relationship of the total integrated scattering from multilayer-coated optics to angle of incidence, polarization, correlation length, and roughness cross-correlation properties," Applied Optics 22, 3207-3219 (1983).

2. C. Amra, "Light scattering from multilayer optics. i. tools of investigation," JOSA A 11, 197-210 (1994).

3. C. Amra, "Light scattering from multilayer optics. ii. application to experiment," JOSA A 11, 211-226 (1994).

4. S. Schröder, T. Herffurth, H. Blaschke, and A. Duparré, "Angle-resolved scattering: an effective method for characterizing thin-film coatings," Applied optics 50, C164-C171 (2011).

5. M. Zerrad, S. Liukaityte, M. Lequime, and C. Amra, "Light scattered by optical coatings: numerical predictions and comparison to experiment for a global analysis," Applied optics 55, 9680-9687 (2016).

6. H. A. Macleod and H. A. Macleod, Thin-film optical filters (CRC press, 2010).

7. P. W. Baumeister, "Optical coating technology," Optimization 10, 7 (2004).

8. C. Deumié, R. Richier, P. Dumas, and C. Amra, "Multiscale roughness in optical multilayers: atomic force microscopy and light scattering," Applied optics 35, 5583-5594 (1996).

9. C. Amra, G. Albrand, and P. Roche, "Theory and application of antiscattering single layers: antiscattering antireflection coatings," Applied optics 25, 2695-2702 (1986).

10. C. Amra, J. H. Apfel, and E. Pelletier, "Role of interface correlation in light scattering by a multiplayer," Applied optics 31, 3134-3151 (1992).

11. M. Demir, J. Johnson, and T. Zajdel, "A study of the fourth-order small perturbation method for scattering from two-layer rough surfaces," Geoscience and Remote Sensing, IEEE Transactions on 50, 33743382 (2012).

12. M. Sanamzadeh, L. Tsang, J. T. Johnson, R. J. Burkholder, and S. Tan, "Scattering of electromagnetic waves from $3 \mathrm{~d}$ multilayer random rough surfaces based on the second-order small perturbation method: energy conservation, reflectivity, and emissivity," JOSA A 34, 395-409 (2017).

13. J.-P. Banon, $\varnothing$. S. Hetland, and I. Simonsen, "Selective enhancement of selényi rings induced by the cross-correlation between the interfaces of a two-dimensional randomly rough dielectric film," Annals of Physics 389, 352-382 (2018).

14. C.-H. Kuo and M. Moghaddam, "Scattering from multilayer rough surfaces based on the extended boundary condition method and truncated singular value decomposition," IEEE Transactions on antennas and propagation 54, 2917-2929 (2006).

15. M. Sanamzadeh, L. Tsang, and J. T. Johnson, "3-d electromagnetic scattering from multilayer dielectric media with 2-d random rough interfaces using $t$-matrix approach," IEEE Transactions on Antennas and Propagation 67, 495-503 (2018).

16. C. Amra, C. Grèzes-Besset, and L. Bruel, "Comparison of surface and bulk scattering in optical multilayers," Applied optics 32, 5492-5503 (1993).

17. T. Elfouhaily and C. A. Guérin, "A critical survey of approximate scattering wave theories from random rough surfaces," Waves in Random Media 14, R1-R40 (2004).

18. L. Tsang, J. A. Kong, K. H. Ding, and C. O. Ao, Scattering of electromagnetic waves: numerical simulations, Wiley series in remote sensing (Wiley-Interscience, 2001).

19. P. Imperatore, A. lodice, M. Pastorino, and N. Pinel, "Modelling scattering of electromagnetic waves in layered media: An up-to-date perspective," International Journal of Antennas and Propagation 2017 (2017).

20. D. Colton and R. Kress, Integral equation methods in scattering theory, vol. 72 (SIAM, 2013).

21. W. Chew, M. Tong, and B. Hu, Integral Equation Methods for Electromagnetic and Elastic Waves (Morgan \& Claypool, 2008).

22. N. Déchamps, N. de Beaucoudrey, C. Bourlier, and S. Toutain, "Fast numerical method for electromagnetic scattering by rough layered interfaces: Propagation-inside-layer expansion method," JOSA A 23, 359-369 (2006).
23. C. Bourlier, N. Pinel, and G. Kubické, Method of moments for $2 D$ scattering problems: basic concepts and applications (John Wiley \& Sons, 2013).

24. J. A. Ogilvy, Theory of Wave Scattering from Random Rough Surfaces (Adam Hilger, Bristol, 1991).

25. G. Hsiao and R. Kleinman, "Mathematical foundations for error estimation in numerical solutions of integral equations in electromagnetics," Antennas and Propagation, IEEE Transactions on 45, 316-328 (1997).

26. J. Zhang, H. Wu, H. Jiao, S. Schröder, M. Trost, Z. Wang, and X. Cheng, "Reducing light scattering in high-reflection coatings through destructive interference at fully correlated interfaces," Optics letters 42, 5046-5049 (2017).

27. J. Zhang, H. Wu, I. V. Kozhevnikov, S. Shi, X. Cheng, and Z. Wang, "Interference suppression of light backscattering through oblique deposition of a layered reflecting coating: bi-layer on a substrate," Optics express 27, 15262-15282 (2019). 